\section{Telehealth and telecommunication in nursing homes during COVID-19 antiepidemic measures in the Netherlands}

Anti-COVID-19 measures for preventing infections in long-term care, such as quarantine and social isolation, revealed the importance of telehealth and telecommunication. Telehealth includes providing health care, treatment, and support through videoconferencing, e-mails, telephone, and apps (Zhou et al., 2020). Our research on challenging behavior during the first pandemic wave in The Netherlands showed initiatives of nursing home staff who provided telehealth with multidisciplinary consultations and support through videoconferencing, e-mail, or telephone. Furthermore, staff facilitated telecommunication during the visitor ban to help residents in staying connected with their loved ones (Leontjevas et al., 2020). To optimize nursing home care in the future, it is important to learn from the experiences with telecommunication and telehealth during the COVID-19 pandemic. For this, we recruited nursing home professionals to fill out an online survey during the second pandemic wave at the end of 2020.

In total, 175 professionals participated, of whom $69(39 \%)$ were psychologists, $61(35 \%)$ therapeutic activities coordinators, 38 (22\%) elderly care physicians or nurse practitioners, and 7 other professionals. The median age was 42 years (quartiles 32, 42,55 years), and the median years of employment in the current organization was 5 years $(2,5,12)$. An overwhelming majority of participants (N/Total valid, valid percentage: $165 / 174,95 \%$ ) confirmed qualitative differences preferring face-to-face contact over telecommunication between residents and their loved ones. However, the value of telecommunication in addition to face-to-face contacts was recognized, and $82 \%(139 / 170)$ of the participants would prefer its continuation after the pandemic. Participants reported challenges in facilitating telecommunication for residents with advanced dementia. Some residents showed negative emotional responses due to poor recognition of relatives or lack of understanding of the situation.

Regarding telehealth and working distantly, a majority of participants (106/165, 64\%; mainly psychologists and physicians or nurse practitioners $[83 / 101,82 \%]$ ) would like to continue at least part of their work remotely after the pandemic. However, more than half of the activities coordinators $(33 / 60,55 \%)$ reported that they did not work remotely during the pandemic while many of them mentioned that it would not be possible given their tasks. Overall, professionals thought that efficiency gains would be possible through telehealth and working distantly. However, although considered workload-reducing, the majority thought that working remotely could worsen their job satisfaction (104/164, 63\%) and the quality of their work $(98 / 169,58 \%)$. Participants expressed the need for more technical support assisting them to work remotely and to facilitate digital communication.

To conclude, the COVID-19 pandemic accelerated the move to and surged the use of telehealth in nursing homes. Yet, for proper implementation, it is necessary to provide a solid infrastructure, to educate and train the staff and residents regarding telecommunication, and to integrate telehealth in daily workflows (Seifert et al., 2020). It is not clear whether and to what extent telehealth can affect outcomes like job satisfaction, the quality of care, and the quality of life of residents. More research into telehealth and telecommunication in nursing homes is needed to help the staff in providing high-quality care.

\section{Conflict of interest}

No conflicts, no sponsors.

\section{References}

Leontjevas, R. et al. (2020). Challenging behavior of nursing home residents during COVID-19 measures in the Netherlands. Aging and Mental Health, 1-6.

Seifert, A., Cotten, S. R. and Xie, B. (2020). A Double Burden of Exclusion?Digital and Social Exclusion of Older Adults in Times of COVID-19. The fournals of Gerontology Series B, Psychological Sciences and Social Sciences.

Zhou, X. et al. (2020). The Role of Telehealth in Reducing the Mental Health Burden from COVID-19. Telemedicine fournal of e-Health, 26, 377-379.

Ruslan Leontjevas, ${ }^{1,2}$ Inge A.H. Knippenberg, ${ }^{1,2}$ Christian BAKkeR, ${ }^{1,3}$

RAYMOND T.C.M. Koopmans ${ }^{1,4}$ and

DebBy L. GerRitsen ${ }^{1}$

${ }^{1}$ Department of Primary and Community Care, Radboud University Medical Centre, Radboud Institute for Health Sciences, Radboudumc Alzheimer Center, Nijmegen, The Netherlands 
${ }^{2}$ Faculty of Psychology, Open University of The Netherlands, Heerlen, The Netherlands

${ }^{3}$ Groenhuysen, Center for Specialized Geriatric Care, Roosendaal, The Netherlands

${ }^{4}$ Joachim en Anna, Centre for Specialized Geriatric Care, Nijmegen, The Netherlands
Correspondence should be addressed to: Ruslan Leontjevas, Faculty of Psychology, Open University of The Netherlands, P.O. Box 2960, 6401 DL Heerlen, The Netherlands. E-mail: roeslan.leontjevas@ou.nl 